\title{
Guideline Recommendations for Oral Care After Acquired Brain Injury: Protocol for a Systematic Review
}

Nalia Gurgel-Juarez ${ }^{1}$, MSc, DDS; Marie-France Perrier ${ }^{1}$, S-LP(C), MHSc; Tammy Hoffmann², BOccThy, PhD; Natasha Lannin ${ }^{3,4,5}$, Grad Cert, Grad Dip, BSc, PhD; Laura Jolliffe ${ }^{3,4}$, B(OT); Rachel Lee ${ }^{1}$, BHSc; Lucie Brosseau ${ }^{1}$, BSc, PhD; Heather Flowers ${ }^{1,6,7,8,9}$, S-LP(C), CCC-SLP, MHSc, MEd, PhD

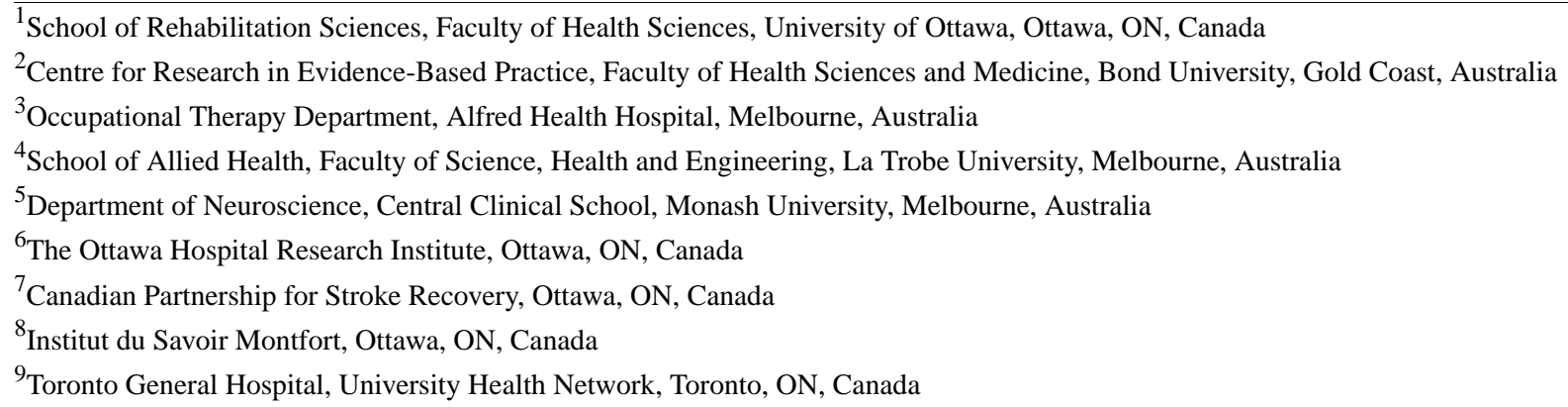

\section{Corresponding Author:}

Nalia Gurgel-Juarez, MSc, DDS

School of Rehabilitation Sciences

Faculty of Health Sciences

University of Ottawa

451 Smyth Rd

Ottawa, ON, K1H 8M5

Canada

Phone: 16135625800 ext 8393

Email: ngurg011@uottawa.ca

\section{Abstract}

Background: Oral care is important to prevent buccal and systemic infections after an acquired brain injury (ABI). Despite recent advancements in the development of ABI clinical practice guidelines, recommendations for specific clinical processes and actions to attain adequate oral care often lack information.

Objective: This systematic review will (1) identify relevant ABI clinical practice guidelines and (2) appraise the oral care recommendations existing in the selected guidelines.

Methods: A search strategy was developed based on a recent systematic review of clinical practice guidelines for ABI. The protocol includes a search of MEDLINE, EMBASE, and DynaMed Plus databases, as well as organizational and best-practice websites and reference lists of accepted guidelines. Search terms will include medical subject headings and user-defined terms. Guideline appraisal will involve the Appraisal of Guidelines for Research and Evaluation II ratings, followed by a descriptive synopsis for oral care recommendations according to the National Health and Medical Research Council evidence levels.

Results: This project started in April 2019, when we developed the search strategy. The preliminary search of databases and websites yielded 863 and 787 citations, respectively, for a total of 1650 citations. Data collection will start in August 2020 and we expect to begin disseminating the results in May 2021.

Conclusions: Nursing staff may not have detailed recommendations on how to provide oral care for neurologically impaired patients. The findings of this review will explore the evidence for oral care in existing guidelines and improve outcomes for patients with ABI. We expect to provide adequate orientations to clinicians, inform policy and guidelines for best practices, and contribute to future directions for research in the ABI realm.

International Registered Report Identifier (IRRID)： PRR1-10.2196/17249

(JMIR Res Protoc 2020;9(7):e17249) doi: 10.2196/17249 


\section{KEYWORDS}

practice guideline; evidence-based recommendations; brain injuries; oral health; oral hygiene; systematic review

\section{Introduction}

Poor oral health may lead to colonization by various microorganisms, causing buccal and respiratory infections [1-5]. Pneumonia, a life-threatening respiratory infection, is a common cause of death after vascular acquired brain injury (ABI) [6] and can be prevented through effective oral hygiene [7-9]. Good oral health is important to preserve one's overall health and well-being [10]. To maintain good oral health, evidence-based oral care practices are essential.

Managing oral care after an acquired brain injury is challenging. The damage that occurs to the brain due to traumatic or nontraumatic causes can lead to physical, behavioral, emotional, and cognitive impairments [11]. For instance, stroke-a nontraumatic, common cause of $\mathrm{ABI}$-leaves one-third of survivors with long-term impairments [12], which may prevent them from managing their oral care. As a result, many are partially or totally reliant on caregivers to maintain their oral hygiene [13]. Nursing staff and family members are usually the ones who provide oral hygiene to care-dependent neurologically impaired survivors [14].

However, clinical barriers may restrict the delivery of high-quality oral care. Insufficient oral care knowledge and training are reported as some of the obstacles for nursing staff to perform adequate care $[15,16]$. Consequently, some professionals feel unprepared $[17,18]$ and prioritize other care activities in the neurosciences context $[19,20]$. In addition, due to an overwhelming work routine in hospitals and long-term care facilities [21-23], nurses sometimes delegate oral care tasks to the least qualified members of the care team [18].

There is little direction from evidence-based literature regarding oral care provision $[18,24,25]$, which results in great variability of practice across the care continuum [26]. Notwithstanding, there have been recent advancements in the development of clinical practice guidelines for ABI, yet they lack specific clinical instructions and processes for adequate and safe oral care provision [27-29]. A comprehensive understanding of existing oral care recommendations in ABI guidelines is badly needed.

The objectives of our proposed systematic review are to (1) update and extend the appraisal of ABI guidelines conducted in a recent systematic review [30], and (2) review and appraise existing oral care recommendations in included guidelines. Since guidelines should ideally be updated every 2 to 3 years [31] and the recent systematic review included ABI guidelines up to 2017 [30], we expect to identify new documents from our updated search. The reasons for extending the search are to help identify literature on oral health across the continuum of care (now including acute settings) and across ABI severity ranges (now including mild ABI). All oral care recommendations will be extracted from the included guidelines and rated according to their levels of evidence. This review will therefore provide a synthesis of best practices for health care professionals, which we anticipate will inform guidelines and practice standards. In addition, our results will provide insight and direction for future research.

\section{Methods}

\section{Scope of the Protocol}

This protocol is adapted from a recent systematic review of clinical practice guidelines for ABI [30]. Our review seeks to synthesize guidelines relevant to a broader range of settings and severities beyond this previous review. Therefore, our search strategy was expanded to include additional relevant information for the acute setting and for mild ABI. Protocol amendments, if necessary, will be incorporated in future publications, with details such as date, description, and rationale of each amendment.

\section{Eligibility Criteria}

Eligible guidelines will include acute and rehabilitation (inpatient and community rehabilitation) settings and pertain to mild to severe ABI. For the purpose of the current protocol, $\mathrm{ABI}$ refers to any damage to the brain that occurs after birth and is not related to a congenital or a degenerative disease [11]. Therefore, causes will include traumatic injury, seizures, tumors, infectious diseases, events in which the brain has been deprived of oxygen, and toxic exposure, such as substance abuse [11].

We will search guidelines published from January 1, 2006, onwards, following the methods of the recent systematic review [30]. Eligible guidelines will be guidelines published in English, particularly those produced under the support of (1) a health professional association or society, (2) a public or private organization, (3) a health care organization or plan, or (4) a government agency [32]. Further, included clinical practice guidelines must contain recommendations, strategies, or information to orient health care professional decisions. Eligible guidelines must have more than 1 component of post-ABI recommendations pertaining to adult patients [27]. In case of incomplete information, we will contact authors of particular guidelines for elaboration or clarification.

\section{Information Sources and Search Strategy}

The protocol combines multiple information sources, including databases as well as organizations and professional websites. Our search will involve MEDLINE, EMBASE, and DynaMed Plus databases. We have developed the search terms for MEDLINE, as seen in Textbox 1, using medical subject headings and user-defined terms. We will apply corresponding terms to the other 2 databases. Subsequently, we will search for organizational and best-practice websites for additional published guidelines. 
Textbox 1. MEDLINE search strategy.

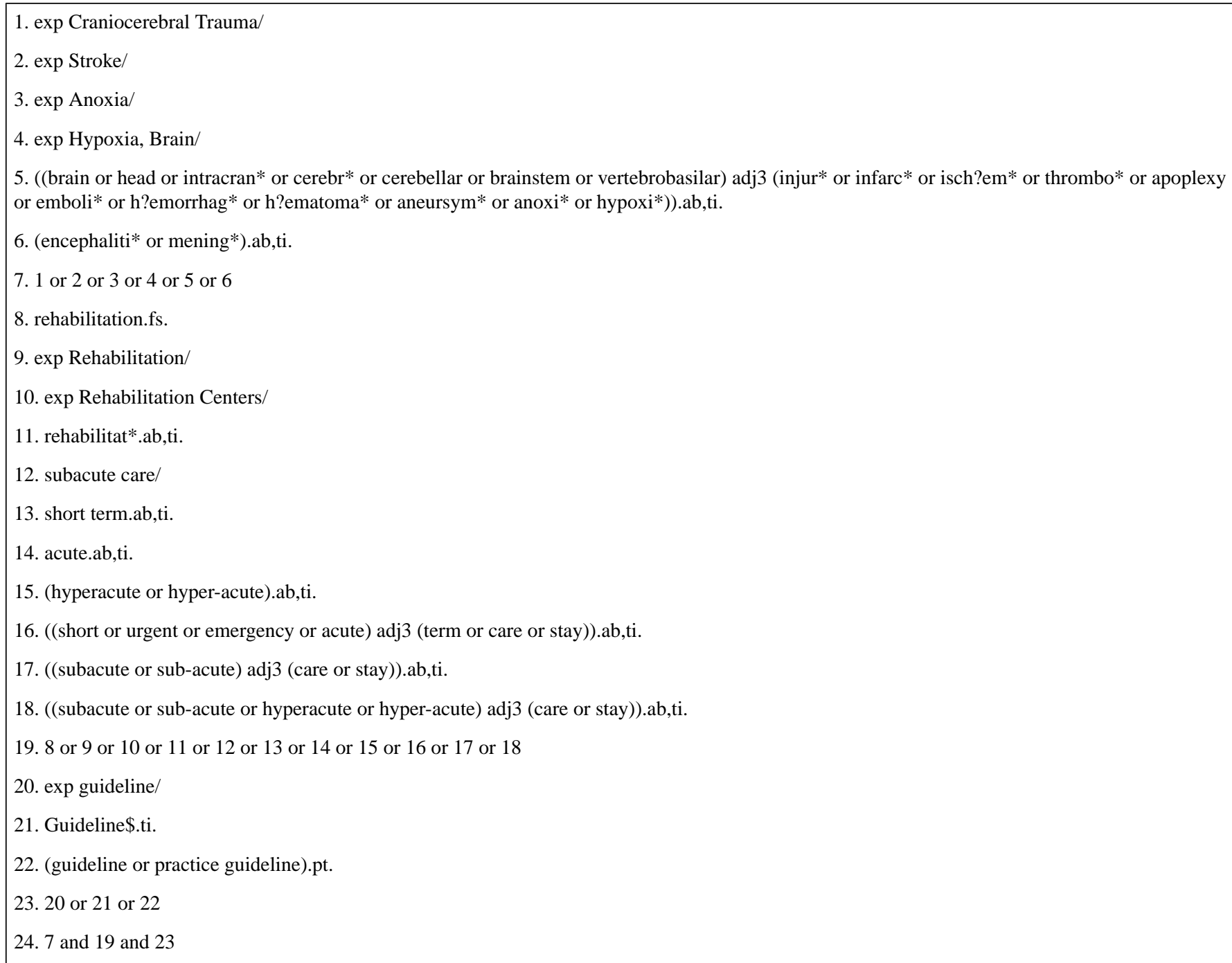

\section{Protocol Guidelines}

The design of this systematic review follows the Preferred Reporting Items for Systematic Review and Meta-Analysis (PRISMA) [33] and PRISMA-Protocols [34,35] guidelines.

\section{Study Records}

\section{Data Management and Collection}

Two independent reviewers (NG-J and M-FP) will use the web-based software Covidence (Veritas Health Innovation Ltd) [36] and the RevMan (Cochrane) software [37] to support and streamline the production of this systematic review. In addition, they will use Excel (Microsoft Corp) spreadsheets to collect, organize, and document excluded and included abstracts and articles.

\section{Selection Process}

A 2-stage process will be used to select included guidelines and extract recommendations. The first stage involves 2 independent reviewers (NG-J and M-FP) reviewing the abstracts of papers that refer to guidelines to identify the guidelines that may be eligible. In the second stage, the same reviewers will evaluate the guidelines in the full articles. These guidelines will be independently reviewed to select those that meet the inclusion criteria. The reviewers will use hierarchical coding criteria to evaluate abstracts and full articles, as shown in Textbox 2. For each stage, they will resolve discrepancies by consensus and, if needed, a third reviewer (HF) will review the documents and contribute to a final consensus decision. 
Textbox 2. Hierarchical coding criteria for abstracts and full articles.

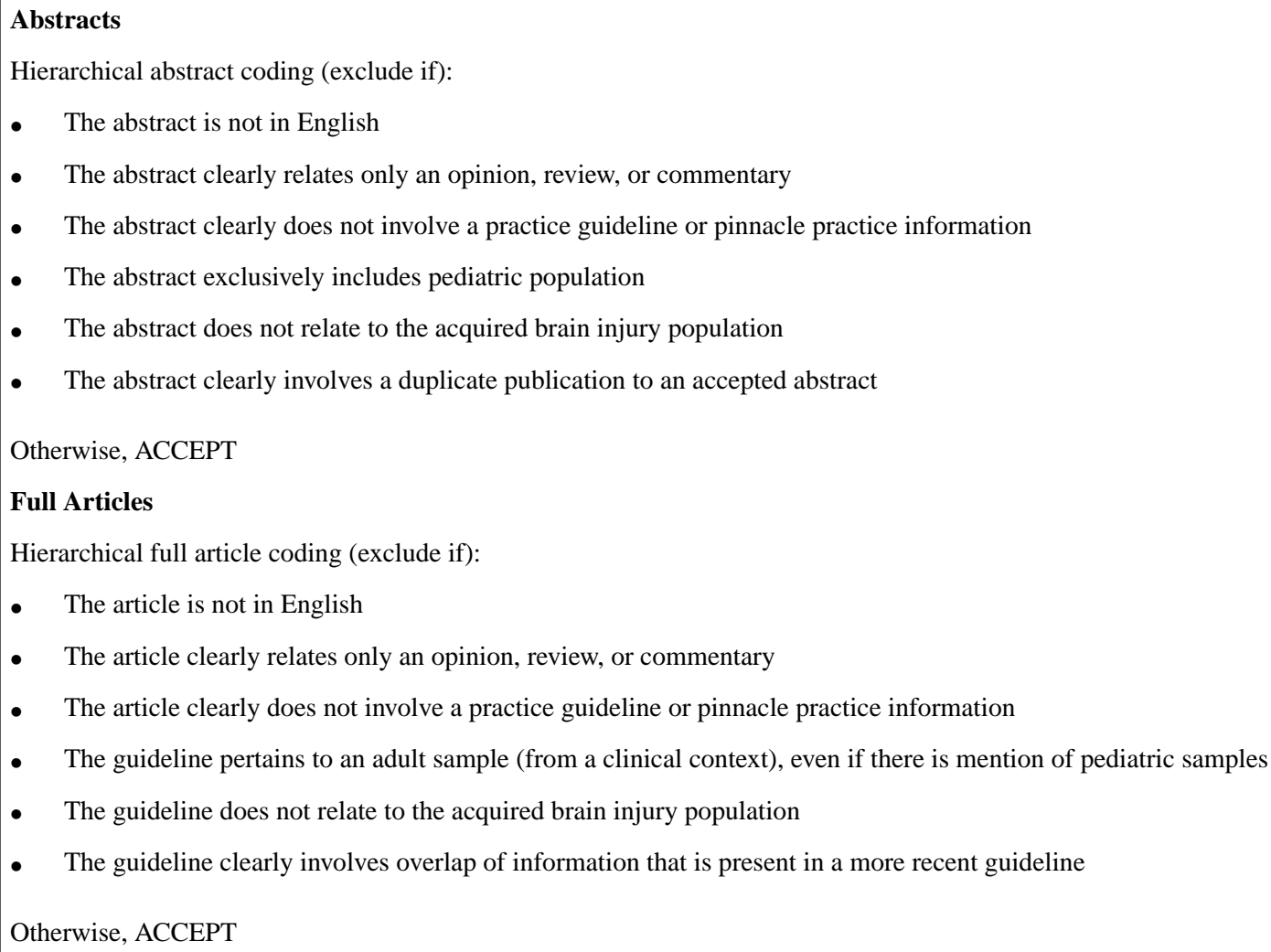

\section{Data Extraction, Appraisal of Guidelines and Recommendations, and Quality Assessment}

Based on our objectives and research questions, data extraction will include the following information: (1) publication specifics (eg, country, organization, and date), (2) setting (eg, acute, rehabilitation, or community), (3) ABI population, (4) target health care professionals, and (5) categories of recommendations.

Two independent authors (LJ and NL) will first evaluate the selected clinical practice guidelines using the Appraisal of Guidelines for Research and Evaluation II (AGREE II) instrument, an international tool used to assess the quality and reporting of practice guidelines [38]. These authors will rate domains of methodological quality for each guideline according to AGREE II. When there is disagreement for a given rating, a third reviewer (TH) will also appraise the guideline with AGREE II.

Subsequently, reviewers NG-J and M-FP will independently evaluate guidelines containing oral care recommendations by using the National Health and Medical Research Council (NHMRC) instrument [39]. This tool allows the assessment and comparison of the levels of evidence and includes grades for the recommendations from the included guidelines. A third reviewer (HF) will resolve disagreements by appraising the recommendations in the same manner. Once the reviewers reach consensus, the 2 initial reviewers (NG-J and M-FP) will provide a descriptive synopsis of the levels of evidence for the oral care recommendations [39]. The risk of bias assessment within and across guidelines and recommendations is included in the Rigour of Development domain of AGREE II and the Evidence Base domain of the NHMRC. Because both tools include risk of bias elements in the quality appraisal, overall quality ratings for the guidelines and recommendations will reflect such information.

\section{Strategy for Data Synthesis}

We will use the AGREE II grades to synthesize the assessed guidelines data, and we will unify the levels of evidence and grades for oral care recommendations according to NHMRC levels to allow comparison and permit description of the evidence. We will retain all identified guidelines and describe their quality and level of evidence. A systematic narrative synthesis will be provided using textual description and tables to summarize and explain the scope, context, and consistency of the clinical practice guideline recommendations. The oral care recommendations will be compared across guidelines to identify similarities and discrepancies. Our systematic review will synthesize data from the guidelines based on quality rankings and levels of evidence for recommendations.

\section{Results}

This project started in April 2019, when we developed the search strategy. The preliminary search of databases and websites yielded 863 and 787 citations, respectively, for a total of 1650 . Data collection will start in August 2020 and we expect to begin disseminating the results in May 2021.

\section{Discussion}

\section{Principal Findings}

Even though good oral care prevents buccal and systemic infections after ABI [40], oral care recommendations are not 
always present in ABI guidelines [27-29]. The provision of oral care can reduce respiratory pathogens in saliva from dental plaque accumulation [41] and residual food or liquid in the oral cavity [42]. Research demonstrates that good oral hygiene decreases pneumonia rates [7] and thereby helps reduce mortality [9], morbidity, and length of hospitalization [6]. In stroke survivors, for instance, pneumonia is responsible for up to $28 \%$ of deaths [43].

However, achieving the means to provide good oral care to neurologically impaired survivors is complex due to physical [44-47], behavioral [48-50], emotional [11], and cognitive impairments [51-53]. Therefore, nursing staff need specific and attainable evidence-based recommendations. Currently, these professionals unfortunately often lack adequate training [14-17], sufficient time [20-22], or satisfactory directions [12,23,24] to provide high-quality oral care, especially in the context of dysphagia [54].

ABI guidelines worldwide endorse oral care for recovery and rehabilitation [27,51-56]. The prevention of pneumonia, notably in patients with dysphagia, is the main reason for recommending oral hygiene [27,40,55,57-59]. However, better functional outcomes, patient comfort, prevention of dental complications, good nutrition, and even prevention of sepsis are also related to mouth care after ABI $[28,40,55,57]$.

\section{Expected Outcomes}

The primary outcomes of this review will include methodological quality of guidelines and levels of evidence for oral care recommendations. We will apply priority rankings to the outcomes to inform health care professionals about highest-quality guidelines and recommendations for oral care, providing direction for future guidelines. The secondary outcome involves developing a best-practice protocol if the evidence is sufficient. There is a great need for a high-quality, process-oriented protocol for provision of oral care in neurologically impaired patients. Such a document could help orient health care professionals in their practice and provide direction for implementation research.

The results of this systematic review will be compared to the full guidelines, and differences between guidelines and recommendations will be explained. Findings may lead to the development of an oral care program that not only delivers safety within the hospital (prevention of complications) but helps clinicians identify opportunities for patients with ABI to independently manage their oral care, improving the long-term outcomes of this population.

\section{Conclusion}

This systematic review will examine the methodological quality of existing recommendations for oral care of patients with ABI. Identifying and appraising the recommendations will support knowledge translation for evidence-based practice across the continuum of care. In addition, it will provide direction for improving future guidelines, developing clinical protocols, and addressing research gaps.

\section{Conflicts of Interest}

None declared.

\section{References}

1. Dai R, Lam OL, Lo EC, Li LS, Wen Y, McGrath C. A systematic review and meta-analysis of clinical, microbiological, and behavioural aspects of oral health among patients with stroke. Journal of Dentistry 2015 Feb;43(2):171-180. [doi: 10.1016/j.jdent.2014.06.005]

2. Corsalini M, Rapone B, Grassi F, Venere DD. A study on oral rehabilitation in stroke patients: analysis of a group of 33 patients. Gerodontology 2010 Sep;27(3):178-182. [doi: 10.1111/j.1741-2358.2009.00322.x] [Medline: 20735492]

3. Yoshida M, Murakami T, Yoshimura O, Akagawa Y. The evaluation of oral health in stroke patients. Gerodontology 2012 Jun;29(2):e489-e493. [doi: 10.1111/j.1741-2358.2011.00505.x] [Medline: 21692834]

4. Anil S, Vellappally S, Hashem M, Preethanath RS, Patil S, Samaranayake LP. Xerostomia in geriatric patients: a burgeoning global concern. J Invest Clin Dent 2014 Sep 01;7(1):5-12. [doi: 10.1111/jicd.12120]

5. Prendergast V, Hinkle JL. Oral Care Assessment Tools and Interventions After Stroke. Stroke 2018 Apr;49(4):e153-e156. [doi: 10.1161/strokeaha.117.017045]

6. Hannawi Y, Hannawi B, Rao CPV, Suarez JI, Bershad EM. Stroke-Associated Pneumonia: Major Advances and Obstacles. Cerebrovasc Dis 2013;35(5):430-443. [doi: 10.1159/000350199]

7. Chan EY, Ruest A, Meade MO, Cook DJ. Oral decontamination for prevention of pneumonia in mechanically ventilated adults: systematic review and meta-analysis. BMJ 2007 Mar 26;334(7599):889. [doi: 10.1136/bmj.39136.528160.be]

8. Fields LB. Oral Care Intervention to Reduce Incidence of Ventilator-Associated Pneumonia in the Neurologic Intensive Care Unit. Journal of Neuroscience Nursing 2008;40(5):291-298. [doi: 10.1097/01376517-200810000-00007]

9. Sjogren P, Nilsson E, Forsell M, Johansson O. A Systematic Review of the Preventive Effect of Oral Hygiene on Hospitals and Nursing Homes: Effect Estimates and Methodological Quality of Randomized Controlled Trials. Journal of American Geriatrics Society 2008;56(11):2124-2130. [doi: 10.1111/j.1532-5415.2008.01926.x]

10. Glick M, Williams DM, Kleinman DV, Vujicic M, Watt RG, Weyant RJ. A new definition for oral health developed by the FDI World Dental Federation opens the door to a universal definition of oral health. Journal of Public Health Dentistry 2017 Mar 09;77(1):3-5. [doi: 10.1111/jphd.12213] 
11. Brain Injury Canada. 2019. Acquired Brain Injury (ABI) - The Basics URL: https://www.braininjurycanada.ca/ acquired-brain-injury/ [accessed 2019-07-16]

12. World Health Organization. 2018. Global Burden of Stroke URL: http://www.who.int/cardiovascular diseases/en/ cvd_atlas_15_burden_stroke.pdf?ua=1 [accessed 2019-06-15]

13. Brady M, Furlanetto D, Hunter R, Lewis S, Milne V. Staff-led interventions for improving oral hygiene in patients following stroke. Cochrane Database Syst Rev 2006 Oct 18(4):CD003864. [doi: 10.1002/14651858.CD003864.pub2] [Medline: 17054189]

14. Cohn JL, Fulton JS. Nursing Staff Perspectives on Oral Care for Neuroscience Patients. Journal of Neuroscience Nursing 2006;38(1):22-30. [doi: 10.1097/01376517-200602000-00006]

15. Kwok C, McIntyre A, Janzen S, Mays R, Teasell R. Oral care post stroke: a scoping review. J Oral Rehabil 2014 Sep 22;42(1):65-74. [doi: 10.1111/joor.12229]

16. Yu P, Fu Q, Shi J, Tao W, Pang H, Chen X, et al. Effects of Different Levels of Caregiver Training on Oral Hygiene After Stroke. J Am Geriatr Soc 2016 Jun 20;64(6):1335-1340. [doi: 10.1111/jgs.14143]

17. Kothari M, Pillai RS, Kothari SF, Spin-Neto R, Kumar A, Nielsen JF. Oral health status in patients with acquired brain injury: a systematic review. Oral Surgery, Oral Medicine, Oral Pathology and Oral Radiology 2017 Feb;123(2):205-219.e7. [doi: 10.1016/j.000o.2016.10.024]

18. Lyons M, Smith C, Boaden E, Brady MC, Brocklehurst P, Dickinson H, et al. Oral care after stroke: Where are we now? European Stroke Journal 2018 May 08;3(4):347-354. [doi: 10.1177/2396987318775206]

19. Wårdh I, Hallberg L, Berggren U, Andersson L, Sörensen S. Oral health care - a low priority in nursing. Scand J Caring Sci 2000;14(2):137-142. [Medline: 12035277]

20. Dickinson H. Maintaining oral health after stroke. Nursing Standard 2012 Aug 08;26(49):35-39. [doi: 10.7748/ns2012.08.26.49.35.c9233]

21. Yoon M, Steele C. Health care professionals' perspectives on oral care for long-term care residents: nursing staff, speech-language pathologists and dental hygienists. Gerodontology 2012 Jun;29(2):e525-e535. [doi: 10.1111/j.1741-2358.2011.00513.x] [Medline: 22462684]

22. Coker E, Ploeg J, Kaasalainen S. The Effect of Programs to Improve Oral Hygiene Outcomes for Older Residents in Long-Term Care: A Systematic Review. Research in Gerontological Nursing 2014 Jan 21;7(2):87-100. [doi: 10.3928/19404921-20140110-01]

23. Odgaard L, Kothari M. Survey of oral nursing care attitudes, knowledge and practices in a neurorehabilitation setting. Journal of Oral Rehabilitation 2019 Apr 21;46(8):730-737. [doi: 10.1111/joor.12799]

24. Talbot A, Brady M, Furlanetto DL, Frenkel H, Williams BO. Oral care and stroke units. Gerodontology 2005 Jun;22(2):77-83. [doi: 10.1111/j.1741-2358.2005.00049.x]

25. Horne M, McCracken G, Walls A, Tyrrell PJ, Smith CJ. Organisation, practice and experiences of mouth hygiene in stroke unit care: a mixed-methods study. J Clin Nurs 2014 Aug 19;24(5-6):728-738. [doi: 10.1111/jocn.12665]

26. Cutler C, Davis N. Improving oral care in patients receiving mechanical ventilation. Am J Crit Care 2005 Sep;14(5):389-394. [Medline: 16120890$]$

27. Hebert D, Lindsay MP, McIntyre A, Kirton A, Rumney PG, Bagg S, et al. Canadian stroke best practice recommendations: Stroke rehabilitation practice guidelines, update 2015. International Journal of Stroke 2016 Apr 14;11(4):459-484. [doi: $10.1177 / 1747493016643553]$

28. Management of Stroke Rehabilitation Working Group. VA/DOD Clinical practice guideline for the management of stroke rehabilitation. J Rehabil Res Dev 2010;47(9):1-43 [FREE Full text] [Medline: 21213454]

29. Stroke Foundation of New Zealand, New Zealand Guidelines Group. Stroke Foundation of New Zealand. 2010. Clinical Guidelines for Stroke Management 2010 URL: https://www.stroke.org.nz/resources/ NZClinicalGuidelinesStrokeManagement2010ActiveContents.pdf [accessed 2019-06-02]

30. Jolliffe L, Lannin NA, Cadilhac DA, Hoffmann T. Systematic review of clinical practice guidelines to identify recommendations for rehabilitation after stroke and other acquired brain injuries. BMJ Open 2018 Feb 28;8(2):e018791. [doi: 10.1136/bmjopen-2017-018791]

31. Vernooij RW, Sanabria AJ, Solà I, Alonso-Coello P, Martínez García L. Guidance for updating clinical practice guidelines: a systematic review of methodological handbooks. Implementation Sci 2014 Jan 2;9(1):3. [doi: 10.1186/1748-5908-9-3]

32. Physioterapy Evidence Database. 2019. Criteria for inclusion of evidence-based clinical practice guidelines URL: https:/ /www.pedro.org.au/english/downloads/criteria/ [accessed 2019-07-20]

33. Liberati A, Altman DG, Tetzlaff J, Mulrow C, Gøtzsche PC, Ioannidis JP, et al. The PRISMA statement for reporting systematic reviews and meta-analyses of studies that evaluate health care interventions: explanation and elaboration. Journal of Clinical Epidemiology 2009 Oct;62(10):e1-e34. [doi: 10.1016/j.jclinepi.2009.06.006]

34. Moher D, Shamseer L, Clarke M, Ghersi D, Liberati A, Petticrew M, et al. Preferred reporting items for systematic review and meta-analysis protocols (PRISMA-P) 2015 statement. Syst Rev 2015 Jan 1;4(1). [doi: 10.1186/2046-4053-4-1]

35. Shamseer L, Moher D, Clarke M, Ghersi D, Liberati A, Petticrew M, et al. Preferred reporting items for systematic review and meta-analysis protocols (PRISMA-P) 2015: elaboration and explanation. BMJ 2015 Jan 02;349(jan02 1):g7647-g7647. [doi: $\underline{10.1136 / \mathrm{bmj} . \mathrm{g} 7647]}$ 
36. Covidence Systematic Review Software. Melbourne: Veritas Health Innovation; 2015. URL: https://www.covidence.org/ home [accessed 2019-07-05]

37. Review Manager (RevMan). Copenhagen: The Cochrane Collaboration; 2014. URL: https://community.cochrane.org/help/ tools-and-software/revman-5 [accessed 2019-07-05]

38. AGREE Next Steps Consortium. 2017. The AGREE II instrument URL: http://www.agreetrust.org [accessed 2018-05-20]

39. National Health and Medical Research Council. 2009. NHMRC levels of evidence and grades for recommendations for developers of guidelines URL: http://citeseerx.ist.psu.edu/viewdoc/ download;jsessionid=AEFFDA62A5245D6D07F060B56789ED5A?doi=10.1.1.177.4984\&rep=rep1\&type=pdf $[$ accessed 2019-05-20]

40. Intercollegiate Stroke Working Party. 2016. National clinical guideline for stroke Internet URL: https://www.strokeaudit.org/ SupportFiles/Documents/Guidelines/2016-National-Clinical-Guideline-for-Stroke-5t-(1).aspx [accessed 2019-02-20]

41. Yoon MN, Steele CM. The Oral Care Imperative. Topics in Geriatric Rehabilitation 2007;23(3):280-288. [doi: 10.1097/01.tgr.0000284771.24711.6a]

42. Cohen DL, Roffe C, Beavan J, Blackett B, Fairfield CA, Hamdy S, et al. Post-stroke dysphagia: A review and design considerations for future trials. International Journal of Stroke 2016 Mar 22;11(4):399-411. [doi: 10.1177/1747493016639057]

43. Westendorp WF, Nederkoorn PJ, Vermeij JD, Dijkgraaf MG, van de Beek D. Post-stroke infection: A systematic review and meta-analysis. BMC Neurology 2011;11:110. [doi: 10.1186/1471-2377-11-110]

44. Pow E, Leung K, Wong M, Li L, McMillan A. A longitudinal study of the oral health condition of elderly stroke survivors on hospital discharge into the community. Int Dent J 2005 Oct;55(5):319-324. [doi: 10.1111/j.1875-595x.2005.tb00330.x] [Medline: 16245468 ]

45. Zhu HW, McMillan AS, McGrath C, Li LSW, Samaranayake LP. Oral carriage of yeasts and coliforms in stroke sufferers: a prospective longitudinal study. Oral Diseases 2008;14(1):60-66. [doi: 10.1111/j.1601-0825.2006.01347.x]

46. Ajwani S, Jayanti S, Burkolter N, Anderson C, Bhole S, Itaoui R, et al. Integrated oral health care for stroke patients - a scoping review. J Clin Nurs 2016 Dec 07;26(7-8):891-901. [doi: 10.1111/jocn.13520]

47. Hunter RV, Clarkson JE, Fraser HW, MacWalter RS. A preliminary investigation into tooth care, dental attendance and oral health related quality of life in adult stroke survivors in Tayside, Scotland. Gerodontology 2006 Sep;23(3):140-148. [doi: 10.1111/j.1741-2358.2006.00125.x]

48. Nijboer T, van de Port I, Schepers V, Post M, Visser-Meily A. Predicting Functional Outcome after Stroke: The Influence of Neglect on Basic Activities in Daily Living. Front. Hum. Neurosci 2013;7:182. [doi: 10.3389/fnhum.2013.00182]

49. Crosby GA, Munshi S, Karat AS, Worthington E, Lincoln NB. Fatigue after stroke: frequency and effect on daily life. Disability and Rehabilitation 2011 Oct 07;34(8):633-637. [doi: 10.3109/09638288.2011.613517]

50. Lerdal A, Gay CL. Acute-Phase Fatigue Predicts Limitations with Activities of Daily Living 18 Months after First-Ever Stroke. Journal of Stroke and Cerebrovascular Diseases 2017 Mar;26(3):523-531. [doi:

10.1016/j.jstrokecerebrovasdis.2016.11.130]

51. Naorungroj S, Slade G, Beck J, Mosley T, Gottesman R, Alonso A, et al. Cognitive Decline and Oral Health in Middle-aged Adults in the ARIC Study. J Dent Res 2013 Jul 19;92(9):795-801. [doi: 10.1177/0022034513497960]

52. Mlinac ME, Feng MC. Assessment of Activities of Daily Living, Self-Care, and Independence. Arch Clin Neuropsychol 2016 Jul 29;31(6):506-516. [doi: 10.1093/arclin/acw049]

53. Foltyn P. Ageing, dementia and oral health. Aust Dent J 2015 Mar 11;60(Suppl 1):86-94. [doi: 10.1111/adj.12287]

54. Stein J, Harvey R, Macko R, Winstein C, Zorowitz R. Stroke recovery \& rehabilitation. New York: Demos Medical Publishing; 2009.

55. Stroke Foundation of Australia. 2017. Clinical Guidelines for Stroke Management 2017 URL: https://informme.org.au/ Guidelines/Clinical-Guidelines-for-Stroke-Management-2017 [accessed 2019-07-08]

56. Scottish Intercollegiate Guidelines Network. 2010. Management of patients with stroke: rehabilitation, prevention and management of complications, and discharge planning, SIGN 118 URL: https://www.sign.ac.uk/ sign-118-management-of-patients-with-stroke-rehabilitation,-prevention-and-management-of-complicati.html [accessed 2019-03-02]

57. Scottish Intercollegiate Guidelines Network. 2010. Management of patients with stroke: identification and management of dysphagia - a national guideline, SIGN 119 URL: https://www.sign.ac.uk/

sign-119-management-of-patients-with-stroke-identification-and-management-of-dysphagia.html [accessed 2019-02-15]

58. National Clinical Guideline Centre. 2013. Stroke Rehabilitation: Long term rehabilitation after stroke - Clinical guideline 162 URL: https://www.ncbi.nlm.nih.gov/books/NBK247494/pdf/Bookshelf_NBK247494.pdf [accessed 2019-08-07]

59. Boulanger J, Lindsay M, Gubitz G, Smith E, Stotts G, Foley N, et al. Canadian Stroke Best Practice Recommendations for Acute Stroke Management: Prehospital, Emergency Department, and Acute Inpatient Stroke Care, 6th Edition, Update 2018. International Journal of Stroke 2018;13(9):949-984. [doi: 10.1177/1747493018786616]

\section{Abbreviations}

ABI: acquired brain injury 
AGREE II: Appraisal of Guidelines for Research and Evaluation II

NHMRC: National Health and Medical Research Council

PRISMA: Preferred Reporting Items for Systematic Review and Meta-Analysis

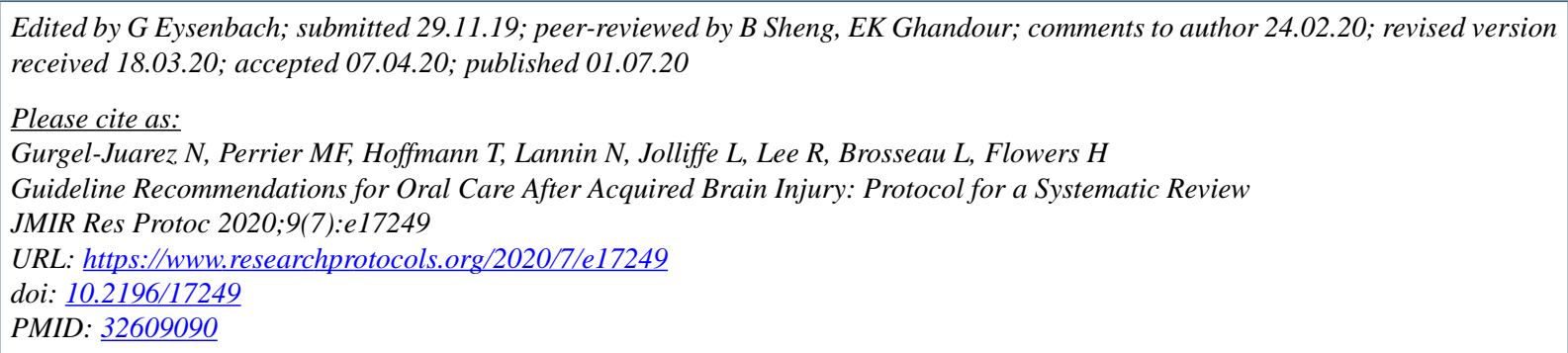

(CNalia Gurgel-Juarez, Marie-France Perrier, Tammy Hoffmann, Natasha Lannin, Laura Jolliffe, Rachel Lee, Lucie Brosseau, Heather Flowers. Originally published in JMIR Research Protocols (http://www.researchprotocols.org), 01.07.2020. This is an open-access article distributed under the terms of the Creative Commons Attribution License (https://creativecommons.org/licenses/by/4.0/), which permits unrestricted use, distribution, and reproduction in any medium, provided the original work, first published in JMIR Research Protocols, is properly cited. The complete bibliographic information, a link to the original publication on http://www.researchprotocols.org, as well as this copyright and license information must be included. 